INVESTIGACIONES

\title{
Diferencias de género y edad en rechazo escolar en una muestra de adolescentes chilenos
}

\author{
Gender and age differences on school refusal in a Chilean adolescents' sample
}

\section{Diferenças de gênero e idade na evasão escolar em uma mostra de adolescentes chilenos}

\section{José Manuel García Fernández, Cándido J. Inglés ${ }^{2}$, Nelly Lagos San Martín³, Carolina Gonzálvez $z^{4}$, María Vicent ${ }^{5}$, María Isabel Gómez Núñez ${ }^{6}$}

${ }^{1}$ Departamento de Psicología Evolutiva y Didáctica, Universidad de Alicante

Tel.: +34965903400 - Ext. 2912, josemagf@ua.es

${ }^{2}$ Facultad de Ciencias Sociosanitarias, Universidad Miguel Hernández de Elche Tel.: +34966658600 - Ext. 9046, cjingles@umh.es

${ }^{3}$ Departamento de Ciencias de la Educación, Universidad del Bío-Bío

Tel.: +56 (42) 46 3483, nlagos@ubiobio.cl

${ }^{4}$ Departamento de Psicología Evolutiva y Didáctica, Universidad de Alicante

Tel.: +34965902492 - Ext. 1210, carolina.gonzalvez@ua.es

${ }^{5}$ Departamento de Psicología Evolutiva y Didáctica, Universidad de Alicante Tel.: +34965902492 - Ext. 1210, maria.vicent@ua.es

${ }^{6}$ Departamento de Psicología Evolutiva y Didáctica, Universidad de Alicante Tel.: +34965903400 - Ext. 2912, marisatobarra11@ hotmail.com

\section{RESUMEN}

El presente estudio tuvo por objeto analizar las diferencias de género y edad en rechazo escolar, en una muestra de adolescentes chilenos. Participaron 2678 estudiantes de Educación Media, 1169 chicos y 1509 chicas, con edades comprendidas entre 13 y 18 años. El rechazo escolar se midió a través de la School Refusal Assessment Scale-Revised for Children (SRAS-R-C), que evalúa el rechazo para evitar situaciones que producen afectividad negativa (Factor I), escapar de situaciones sociales y/o evaluativas (Factor II), llamar la atención de personas significativas (Factor III) y obtener recompensas tangibles fuera del colegio (Factor IV). Los resultados derivados del análisis de varianza revelaron que las chicas puntuaron significativamente más alto que los chicos en el Factor I, mientras que los chicos puntuaron significativamente más alto que las chicas en los Factores II y IV. Asimismo, se hallaron diferencias de edad en los cuatro factores que componen el rechazo escolar.

Palabras claves: rechazo escolar, adolescencia, diferencias de género, diferencias de edad.

\section{ABSTRACT}

The aim of this study was to analyze gender and age differences on school refusal in a Chilean adolescents' sample. 2678 Middle School students, 1169 boys and 1509 girls, between 13 and 18 years old participated in our research. School refusal was assessed by the implementation of the School Refusal Assessment Scale-Revised for Children (SRAS-R-C), which measures the refusal to avoid school-based stimuli provoking negative affectivity (Factor I), escape from aversive social and/or evaluative situations (Factor II), attention seeking from significant people (Factor III), and pursue of tangible rewards outside of school (Factor IV). Results derived from analysis of variance revealed that the girls scored notably higher than the boys in factor I, while the boys scored notably higher than the girls in factors II and IV. Additionally, age differences were found for the four factors that integrate school refusal.

Keywords: school refusal, adolescence, gender differences, age differences. 


\section{RESUMO}

O presente estudo teve como objetivo analisar as diferenças de acordo com sexo e idade na evasão escolar em uma mostra de adolescentes chilenos. Participaram 2.678 estudantes do Ensino Fundamental e Médio, 1.169 alunos e 2.678 alunas, com idade entre 13 e 18 anos. A evasão escolar foi medida através da School Refusal Assessment Scale-Revised for Children (SRAS-R-C) que a avalia para evitar situações que produzam afetividade negativa (Fator I), escapar de situações sociais e/ou avaliativas (Fator II), chamar a atenção de pessoas significativas (Fator III) e obter recompensas tangíveis fora da escola (Fator IV). Os resultados da análise de variância revelaram diferenças significativas para o Fator I, em nome das alunas, e para os Fatores II e IV, em favor dos alunos. Também foram encontradas diferenças de idade entre os quatro fatores que compõem o abandono escolar.

Palavras chave: abandono escolar, adolescência, diferenças de gênero, diferenças de idade.

\section{INTRODUCCIÓN}

\subsection{RECHAZO ESCOLAR}

El rechazo escolar es un constructo amplio, referido a la negativa de un niño a asistir a la escuela o la dificultad persistente para permanecer en ella (Kearney y Bates 2005). Así, el término de rechazo a la escuela es un concepto global que subsume los distintos tipos de inasistencia a la escuela: absentismo escolar, fobia escolar, ansiedad escolar o la negativa a asistir a la escuela con base ansiógena ante la separación de una figura afectiva (Kearney, 2007).

En la literatura científica se establece una diferenciación entre los dos principales tipos de niños y/o jóvenes con rechazo escolar. Por un lado, se encuentran aquellos sujetos que rechazan la escuela en base a la ansiedad generada o debido al miedo causado por la institución educativa, el cual suele producirse bajo el consentimiento paterno, mientras que aquellos identificados como absentistas basan su negativa a asistir a la escuela en el aburrimiento asociado a la escuela, la preferencia de estar con amigos en la calle o jugando en casa, la manifestación de conductas antisociales o delictivas, siendo este un comportamiento que suele producirse sin la autorización familiar (Kearney, 2008; Thomas et al. 2011; Wilkins, 2008; Wimmer, 2008).

Kearney, Turner y Gauger (2010) caracterizan a los jóvenes con rechazo escolar de tipo leve como jóvenes que faltan esporádicamente a la escuela, cuya inasistencia se produce en algunas clases, que llegan tarde o que se van antes de terminar la jornada escolar, logrando tales comportamientos a partir de quejas, coacción o súplicas. Por otro lado, se encuentran los jóvenes que se ausentan totalmente del colegio durante un período prolongado de tiempo, tratándose de un rechazo escolar grave (King, Ollendick y Tonge, 1995). Kearney (2007) indica que si la problemática de rechazo perdura durante dos semanas, sería considerado de tipo agudo, mientras que si se extiende durante más de un año, el rechazo escolar sería de tipo crónico.

El modelo funcional es el principal enfoque de clasificación de la conducta de rechazo a la escuela, en base a los cuatro factores o razones que subyacen a este comportamiento: evitar la afectividad negativa que provocan los estímulos o situaciones relacionadas con el ámbito escolar, escapar de la aversión social o situaciones de evaluación, búsqueda de la atención de otras personas significativas y búsqueda de refuerzos tangibles fuera del ámbito escolar (Kearney y Silverman, 1996). En base a este postulado teórico, se ha diseñado el único instrumento específico de evaluación del rechazo escolar dirigido a niños, 
adolescentes y padres, la School Refusal Assessment Scale-Revised (SRAS-R; Kearney, 2002). La categorización propuesta por este modelo no implica necesariamente que en el origen del rechazo escolar haya una única razón, sino que también puede suceder que se deba a dos o más condiciones funcionales simultáneas o progresivas (Kearney, Lemos y Silverman, 2004). Por ejemplo, el caso de un niño que inicialmente rechaza la escuela para evitar estímulos aversivos y que, posteriormente, puede modificar o incrementar sus razones para rechazar la escuela (Kearney, 2007).

El rechazo escolar es un fenómeno que presenta una alta comorbilidad con otras problemáticas, tales como la ansiedad ante la separación de los padres, el trastorno de ansiedad generalizada, trastorno oposicionista desafiante, acontecimientos relacionados con la escolaridad (ser objeto de burlas, hablar delante de los compañeros, ser enviado al director, relación con el docente, hacer exámenes) o depresión (Kearney y Albano, 2004; Gastaldi et al. 2014). La conducta negativa a asistir a la escuela conlleva a una serie de consecuencias significativas y adversas en el desarrollo del niño afectado, señalándose su influencia en un bajo rendimiento académico, conflictos familiares, problemas sociales, malestar físico (Kearney, 2001) e incluso llegar a la deserción escolar (Teasley, 2004).

Determinar con exactitud la prevalencia del rechazo escolar resulta difícil debido a la imprecisión de los criterios de clasificación que han predominado para su estudio y la falta de informes estandarizados. Cuantificar el porcentaje de escolares afectados por esta problemática es una tarea compleja en la que se debe distinguir entre contabilizar sólo los días completos que un estudiante pierde en un año lectivo o aquellos casos en los que ha llegado tarde a la escuela, se ha ido antes a casa o simplemente porque ha evadido algunas de las clases durante el día. A pesar de estas limitaciones, las tasas de prevalencia de esta problemática se encuentran en torno al 5\% de los niños en edad escolar (Kearney, 2001; Sewell, 2008), mientras que el comportamiento de rechazo a la escuela entendido como el constructo conceptual que engloba los distintos grados de inasistencia (retrasos, conducta negativa a asistir a la escuela, faltas esporádicas a clases, faltas crónicas a clases) afecta a entre un $28 \%$ y $35 \%$ de los jóvenes en algún momento de sus vidas (Mihalas, 2014). Además, estudios señalan la existencia de períodos temporales en los que prevalece una mayor tasa de incidencia de esta conducta, encontrando divergencias entre los resultados en función del sexo y la edad (Fremont, 2003; Guare y Cooper, 2003; Kearney y Spear, 2014), controversias que pueden deberse a las diferencias encontradas en la conceptualización del rechazo escolar y sus criterios de clasificación (Kearney y Graczyk, 2014).

\subsection{DIFERENCIAS DE GÉNERO EN RECHAZO ESCOLAR}

En cuanto al estudio de las diferencias de género en rechazo escolar, predominan los trabajos que indican que su distribución en función del sexo prevalece de manera equilibrada en chicos y chicas (Fremont, 2003; Guare y Cooper, 2003; Wijetunge y Lakmini 2011). Sin embargo, diversos estudios refutan esta idea, señalando que el rechazo a la escuela basado en la ansiedad generada ante la separación de un ser querido o figura de referencia es superior en chicas (Fan, Su y Su, 2008; Gosch, Flannery-Schroeder y Brecher, 2012), mientras que generado por una fobia específica resulta predominante en chicos (King, Ollendick y Tonge, 1995). Estudios posteriores plantean que las tasas de deserción escolar son ligeramente superiores en chicos que en chicas (Kearney, 2008; Kearney y Spears, 2014). 


\subsection{DIFERENCIAS DE EDAD EN RECHAZO ESCOLAR}

En relación con la edad, los estudios han demostrado que se trata de una problemática que puede comenzar en cualquier momento. No obstante, en los niños pequeños, el inicio tiende a ser repentino y de tipo agudo, mientras que en los preadolescentes y adolescentes, el desarrollo es más gradual y más grave, sobre todo porque en estas etapas el rechazo escolar tiene una mayor comorbilidad con trastornos como la depresión y la ansiedad (Heyne et al. 2011). La conducta de rechazo a la escuela presenta mayor incidencia en determinados momentos educativos que suponen una mayor probabilidad de desarrollar rechazo, tales como el inicio del curso académico, el cambio a un nuevo centro escolar o la transición a otra etapa educativa (Kearney y Albano, 2004; Kearney y Bates, 2005; Pina et al. 2009). Esta situación es corroborada por Wijetunge y Lakmini (2011), quienes suman a los momentos de inicio escolar y cambio de escuela, la etapa pre-adolescente.

En términos de grupos etarios, Ollendick y Mayer (1984) señalan una mayor incidencia en los grupos de 5-6 años y 10-11,5 años. Tolin et al. (2009) amplían estos grupos, incorporando los chicos de 13-14 años como especialmente sensibles al rechazo escolar. A juicio de Kearney, Lemos y Silverman (2006), el rechazo escolar es ampliamente presentado por chicos entre 10 y 13 años, rango etario que abarca el alumnado que supera la Educación Básica, y además, señalan el ingreso a una nueva escuela como hecho que puede afectar a cualquier edad.

Con el propósito de paliar las discrepancias identificadas, es objeto del presente estudio analizar las diferencias de género y edad en rechazo escolar en una muestra representativa de adolescentes chilenos. De este modo, y teniendo en consideración los hallazgos de los estudios previos, se espera que los chicos presenten puntuaciones más altas de rechazo no basado en la ansiedad, debido a su mayor vinculación con la deserción escolar, mientras que las chicas presentarán puntuaciones más altas en rechazo a la escuela en función de la ansiedad generada ante la separación de una figura significativa. Respecto a la edad, se espera que el grupo entre 13 y 14 años presente puntuaciones elevadas en rechazo escolar en comparación con los grupos 15-16 y 17-18 años, debido a que se encuentran en un período de transición educativa, es decir, el paso de la Educación Básica a la Educación Media.

\section{MÉTODO}

\subsection{PARTICIPANTES}

La investigación se realizó en 25 centros educativos de Educación Media localizados en 7 comunas de la provincia de Nuble en Chile, seleccionados al azar y siendo, aproximadamente, un centenar el número de participantes por centro. El número total de la muestra estuvo compuesto por 2.746 estudiantes, de los cuales 68 fueron excluidos debido a errores u omisiones en sus respuestas o por no haber obtenido el consentimiento de sus padres para participar en la investigación. La muestra final incluyó un total de 2.678 estudiantes, 1.169 chicos y 1.509 chicas (43,7\% y 56,3\%, respectivamente), cuyo rango de edad osciló entre los 13 y 18 años $(M=16, D T=1.35)$. Utilizando la prueba Chi-cuadrado de homogeneidad de la distribución de frecuencias, se comprobó que no existían diferencias 
estadísticamente significativas entre los seis grupos de Género x Curso $\left(\mathrm{c}^{2}=.43 ; p=.81\right)$. La Tabla 1 presenta la distribución de la muestra por género y edad.

Tabla 1. Distribución de frecuencias (y porcentajes) Género x Edad

\begin{tabular}{|l|c|c|c|c|}
\hline & $13-14$ años & $15-16$ años & $17-18$ años & Total \\
\hline Chicos & $349(13.0 \%)$ & $582(21.7 \%)$ & $238(08.9 \%)$ & $1.169(43.7 \%)$ \\
\hline Chicas & $463(17.3 \%)$ & $732(27.3 \%)$ & $314(11.7 \%)$ & $1.509(56.3 \%)$ \\
\hline Total & $812(30.3 \%)$ & $1.314(49.1 \%)$ & $552(20.6 \%)$ & $2.678(100 \%)$ \\
\hline
\end{tabular}

\subsection{INSTRUMENTO}

Para medir el rechazo escolar se aplicó la adaptación chilena (Lagos-San Martín et al. 2014) de la versión para niños de la School Refusal Assessment Scale-Revised (SRAS-R; Kearney, 2002). Este instrumento mide el rechazo escolar en niños y adolescentes de entre 6 y 19 años de edad y contiene 24 preguntas que se responden en una escala likert de 7 puntos, que va desde 0 (nunca) hasta 6 (siempre), a partir de 4 dimensiones: I) evitación de estímulos o situaciones relacionadas con el contexto escolar que provocan afectividad negativa, II) escape de situaciones sociales y/o evaluativas aversivas, III) búsqueda de atención de personas significativas y IV) búsqueda de reforzamiento positivo fuera del colegio. La obtención de una puntuación más alta en uno o más factores es indicador de un mayor rechazo escolar y se considerará a ese o esos factores como los principales causantes de su mantenimiento.

Los coeficientes de consistencia interna (alfa de Cronbach) en el presente estudio fueron de .75 (Factor I), .78 (Factor II), .76 (Factor III) y .74 (Factor IV), los cuales son muy similares a la versión original.

\subsection{PROCEDIMIENTO}

Se llevó a cabo una entrevista con los directores de los centros seleccionados para exponer los objetivos de la investigación, obtener la autorización y acordar un horario para la aplicación del instrumento. Igualmente, se solicitó el consentimiento de los padres para participar en la investigación. Durante el encuentro con los estudiantes, se dieron las instrucciones sobre la forma de responder, enfatizando la importancia de no dejar ítems sin contestar. Se realizó un ejercicio colectivo a modo de ejemplo y a partir de ahí los estudiantes contestaron. Uno de los investigadores estuvo presente durante toda la aplicación para proporcionar ayuda, verificar la cumplimentación correcta e independiente por parte de los sujetos, asegurándose de que los datos de identificación fueran debidamente registrados. Una vez completadas las pruebas, se agradeció la participación y la colaboración en el proyecto a cada uno de los grupos de manera conjunta (alumnado, docentes y equipo directivo), asegurando la entrega de un informe con los resultados obtenidos y con sugerencias educativas de apoyo. 


\subsection{ANÁLISIS DE DATOS}

Para analizar las diferencias de sexo y edad en rechazo escolar, se realizaron análisis de varianza (ANOVAs). Además, para concretar entre qué grupos de edad se daban las diferencias, se utilizó la prueba post-hoc de Scheffé. Con objeto de estimar la magnitud de las diferencias encontradas, se calcularon los tamaños del efecto mediante el índice $d$ (diferencia de media tipificada) de Cohen (1988), el cual fue interpretado de la siguiente manera: magnitudes bajas (entre .20 y .49), moderadas (entre .50 y.79) y altas (superiores a .80).

\section{RESULTADOS}

\subsection{DIFERENCIAS DE GÉNERO EN RECHAZO ESCOLAR}

Los resultados del ANOVA mostraron diferencias estadísticamente significativas entre ambos sexos para el FI, FII y FIV. En el caso del FI, los chicos presentaron significativamente puntuaciones más bajas que sus iguales del sexo opuesto. Por el contrario, las chicas presentaron puntuaciones más bajas en los factores II y IV que sus compañeros. Los tamaños del efecto fueron pequeños en todos los casos (véase la Tabla 2).

Tabla 2. Diferencias según el género

\begin{tabular}{|l|c|c|c|c|c|c|c|}
\hline & \multicolumn{2}{|c|}{ Chicos } & \multicolumn{2}{c|}{ Chicas } & \multicolumn{2}{c|}{ Significación estadística y magnitud de las diferencias } \\
\hline & $\mathrm{M}$ & $\mathrm{DE}$ & $\mathrm{M}$ & $\mathrm{DE}$ & $F_{(1,1412)}$ & $p$ & $d$ \\
\hline FI & 14.62 & 6.52 & 15.09 & 6.56 & 4.29 & .03 & .07 \\
\hline FII & 13.46 & 6.22 & 12.81 & 5.55 & 7.63 & $<.001$ & .11 \\
\hline FIII & 17.65 & 7.33 & 17.65 & 7.24 & .02 & .87 & - \\
\hline FIV & 23.69 & 7.07 & 22.22 & 6.83 & 27.21 & $<.001$ & .21 \\
\hline
\end{tabular}

Nota. FI = Rechazo para evitar emociones negativas; FII = Rechazo para escapar de situaciones sociales y/o evaluativas aversivas; FIII = Rechazo para llamar la atención de los demás; FIV = Rechazo para obtener recompensas tangibles fuera de la escuela.

\subsection{DIFERENCIAS DE EDAD EN RECHAZO ESCOLAR}

Tal y como se presenta en la Tabla 3, se hallaron diferencias de edad estadísticamente significativas en los cuatro factores que componen el rechazo escolar. Concretamente, la prueba Scheffé reveló que los alumnos entre 13 y 14 años puntuaron significativamente más bajo en el Factor I del rechazo escolar que sus compañeros entre 15 y 16 años $(d=.26)$ y entre 17 y 18 años $(d=.28)$. Igualmente, los participantes entre 13 y 14 años obtuvieron puntuaciones significativamente menores en el Factor II que sus iguales entre 15 y 16 años 
$(d=.12)$. Respecto al Factor III, los estudiantes de menor edad puntuaron más bajo que los escolares entre 15 y 16 años $(d=.10)$, y éstos, a su vez, obtuvieron puntuaciones más altas que sus iguales entre 17 y 18 años $(d=.11)$. Además, los estudiantes entre 13 y 14 años puntuaron significativamente más bajo en el Factor IV que sus compañeros entre 15 y 16 años $(d=.14)$.

Tabla 3. Diferencias según la edad

\begin{tabular}{|l|c|c|c|c|c|c|c|c|}
\hline & \multicolumn{2}{|c|}{$13-14$ años } & \multicolumn{2}{c|}{$15-16$ ã̃os } & \multicolumn{2}{c|}{$17-18$ años } & \multicolumn{2}{c|}{ Significación estadística } \\
\hline & $\mathrm{M}$ & $\mathrm{DE}$ & $\mathrm{M}$ & $\mathrm{DE}$ & $\mathrm{M}$ & $\mathrm{DE}$ & $F_{(2,1411)}$ & $p$ \\
\hline FI & 13.69 & $(6.31)$ & 15.37 & $(6.56)$ & 15.50 & $(6.62)$ & 18.95 & $<.001$ \\
\hline FII & 12.65 & $(5.42)$ & 13.33 & $(6.03)$ & 13.20 & $(6.02)$ & 3.68 & .02 \\
\hline FIII & 17.30 & $(7.24)$ & 18.04 & $(7.31)$ & 17.25 & $(7.21)$ & 3.54 & .02 \\
\hline FIV & 22.25 & $(6.82)$ & 23.19 & $(6.97)$ & 22.96 & $(7.14)$ & 5.59 & $<.001$ \\
\hline
\end{tabular}

Nota. FI = Rechazo para evitar emociones negativas; FII = Rechazo para escapar de situaciones sociales y/o evaluativas aversivas; FIII = Rechazo para llamar la atención de los demás; FIV = Rechazo para obtener recompensas tangibles fuera de la escuela.

No se encontró un efecto estadísticamente significativo para la interacción Género $\mathrm{x}$ Edad en los 4 factores que componen el rechazo escolar.

\section{CONCLUSIONES}

El objetivo de este estudio fue analizar las diferencias de género y curso en rechazo escolar, mediante el instrumento de evaluación SRAS-R, en una muestra representativa de adolescentes chilenos.

En cuanto a las diferencias de género, los resultados indicaron la existencia de diferencias significativas en tres de los cuatro factores evaluados, aunque de pequeña magnitud. Por un lado, los chicos obtuvieron puntuaciones significativamente superiores en los factores II y IV que las chicas, apoyando parcialmente nuestra hipótesis inicial según la cual los chicos presentarían puntuaciones más altas para el factor IV, debido a su fundamentación no basada en la ansiedad ante el rechazo y dada su mayor vinculación con la deserción escolar, fenómeno más común en el género masculino, tal y como corroboran estudios previos (Brill, 2009; Kearney, 2008; Kearney y Spears, 2014). En cuanto a los resultados obtenidos para el factor II, el género masculino presenta mayores puntuaciones de rechazo escolar que las chicas, en consonancia con el estudio de King, Ollendick y Tonge (1995), quienes señalan mayor vinculación de los chicos con problemas de índole social y de evaluación. Por otro lado, se halló que las chicas obtuvieron una media significativamente superior a los hombres en el factor I, en coherencia con las indicaciones señaladas por Brill (2009), en las que las 
causas de rechazo en chicas se encuentran más alineadas con la ansiedad, las fobias y las perturbaciones emocionales que en el caso de los chicos. Sin embargo, contrariamente a lo esperado, las chicas no puntuaron más alto que los chicos en el Factor III, para el que no se encontraron diferencias estadísticamente significativas. Este resultado puede deberse a que la teoría revela puntuaciones más altas para chicas en este factor pero en edad infantil, etapa en la que la separación de una figura querida provoca niveles superiores de rechazo escolar en el género femenino (Compton, Nelson y March, 2000; Dent y Cameron, 2003; Fan, Su y Su, 2008; Gosch, Flannery-Schroeder y Brecher, 2012), no cumpliéndose en nuestro estudio dicha relación en la muestra adolescente.

En relación a las diferencias encontradas según la edad, los resultados indicaron una tendencia de los estudiantes entre 15 y 16 años a obtener puntuaciones más altas en rechazo a la escuela que sus iguales de mayor y menor edad, en contraposición con lo esperado, según las investigaciones previas que planteaban la etapa de transición educativa, en este caso alrededor de los 14 años (Etapa de Educación Básica-Etapa de Educación Media), como un momento de mayor incidencia (Kearney, Lemos y Silverman, 2006). No obstante, la literatura científica indica que el fenómeno del rechazo escolar puede generarse en cualquier momento, siendo la etapa de la adolescencia uno de los momentos de mayor vulnerabilidad (Wijetunge y Lakmini, 2011) y situándose el grupo etario entre 15 y 16 años en una nueva etapa educativa del sistema escolar chileno.

En términos de deserción escolar prematura, en Chile se ha encontrado que el 34,3\% de los estudiantes que no han completado la enseñanza media justifica su abandono escolar en el desinterés por estudiar, ya que consideraban que era una actividad que no les gustaba (MIDEPLAN, 2005). Es por ello que se precisa de una detección temprana de esta problemática desde el ámbito educativo (Teasley, 2004), ya que el absentismo es uno de los principales indicadores que pueden conllevar al abandono escolar y sus consecuencias negativas para el desarrollo académico, social y personal del joven afectado (Epstein y Sheldon, 2002).

Este estudio presenta varias limitaciones que sería recomendable tener en consideración para futuras investigaciones sobre la temática que nos ocupa. En primer lugar, puesto que los resultados obtenidos en este trabajo han sido obtenidos mediante un diseño transversal, sería importante utilizar diseños longitudinales para futuras investigaciones con objeto de evaluar las tendencias de cambio intra-sujeto. En segundo lugar, sería conveniente emplear otros instrumentos o entrevistas de evaluación del rechazo escolar dirigidas no sólo a niños, sino también a docentes o padres, que permitan dotar de mayor rigor la información obtenida, utilizando distintos tipos de cuestionarios o autoinformes de pregunta abierta. Además, sería conveniente ampliar las edades estudiadas, para observar las fluctuaciones en las puntuaciones de rechazo escolar durante la infancia tardía (6 a 12 años), ya que son diversos los estudios encontrados que analizan los índices de deserción escolar, pero centrándose en la población universitaria chilena (Díaz-Peralta, 2008; FernándezGonzález, Martínez-Conde Beluzan y Melipillán-Araneda, 2009). No obstante, tal y como señala Teasley (2004), el comportamiento de rechazo a la escuela puede desarrollarse progresivamente alcanzando la deserción en niveles superiores, por lo que podría resultar una implicación educativa de relevancia la detección e intervención durante la etapa de Educación Básica, favoreciendo así la atención de estos sujetos afectados por el rechazo a la escuela en edades más tempranas y evitando, consecuentemente, la consolidación de esta conducta. 
A pesar de estas limitaciones, los hallazgos de esta investigación aportan a la comunidad científica las diferencias de género y edad en rechazo escolar encontradas en una muestra representativa de población adolescente chilena, siendo una contribución relevante para el avance científico en el ámbito del rechazo escolar durante la etapa de Educación Media y su estudio pionero en esta zona geográfica.

\section{REFERENCIAS BIBLIOGRÁFICAS}

Brill, L. D. (2009). School Refusal: Characteristics, Assessment, and Effective Treatment: A Child and Parent Perspective (Tesis doctoral). Recuperado el 22 de octubre de 2014 de http://digitalcommons.pcom.edu/cgi/viewcontent.cgi?article=1016\&context=psychology_ dissertations

Cohen, J. (1988). Statistical power analysis for the behavioral sciences. Hillsdale: Lawrence Erlbaum Associates, Publishers.

Compton, S. N., Nelson, A. H. y March, J. S. (2000). Social phobia and separation anxiety symptoms in community and clinical samples of children and adolescents. Journal of the American Academy of Child and Adolescent Psychiatry, vol.39, n.8, 1040-1046. doi: 10.1097/000045832000080000-00020

Dent, R. J. y Cameron, R. J. S. (2003). Developing resilience in children who are in public care: The educational psychology perspective. Educational Psychology in Practice, vol.19, n.1, 3-19. doi: $10.1080 / 026673603200061170$

Díaz-Peralta, C. (2008). Modelo conceptual para la deserción estudiantil universitaria chilena. Estudios pedagógicos, vol.34, n.2, 65-86.

Epstein, J. L, y Sheldon, S. B. (2002). Present and Accounted for: Improving Student Attendance through Family and Community Involvement. Journal of Education Research, vol.95, n.5, 308318. doi: 10.1080/00220670209596604

Fan, F., Su. L. y Su, Y. (2008). Anxiety structure by gender and age groups in a Chinese children sample of 12 cities. Chinese Mental Health Journal, n.22, 241-245.

Fernández-González, O. M., Martínez-Conde Beluzan, M. y Melipillán-Araneda, R. (2009). Estrategias de aprendizaje y autoestima: su relación con la permanencia y deserción universitaria. Estudios pedagógicos, vol.35, n.1, 27-45.

Fremont, W. P. (2003). School refusal in children and adolescents. American Family Physician, n.68, $1555-1561$.

Gastaldi, F. G. M., Pasta, T., Longobardi, C., Prino, L. E. y Quaglia, R. (2014). Measuring the influence of stress and burnout in teacher-child relationship. European Journal of Education and Psychology, vol.4, n.1, 17-28. doi: 10.1989/ejep.v7i1.149

Gosch, E. A., Flannery-Schroeder, E. y Brecher, R. J. (2012). Anxiety Disorders School-Based Cognitive-Behavioral Interventions. En R. B. Mennutti, A. Freeman y R.W. Christner (Eds.), Cognitive-behavioral interventions in educational settings: A handbook for practice (pp. 117160). New York: Taylor \& Francis.

Guare, R. E., y Cooper, B. S. (2003). Truancy revisited: Students as school consumers. Washington, DC: American Psychological Association.

Heyne, D., Sauter, F.M., Van Widenfelt, B.M., Vermeiren, R. y Westenberg, P. M. (2011). School refusal and anxiety in adolescence: Non-randomized trial of a developmentally sensitive cognitive behavioral therapy. Journal of Anxiety Disorders, vol.25, n.7, 870-878. doi: 10.1016/j. janxdis.2011.04.006

Kearney, C. A. (2008). School absenteeism and school refusal behavior in youth: A contemporary review. Clinical Psychology Review, vol.28, n.3, 451-471. doi: 10.1016/j.cpr.2007.07.012 
Kearney, C. A. (2007). Forms and functions of school refusal behavior in youth: An empirical analysis of absenteeism severity. Journal of Child Psychology and Psychiatry, vol.48, n.1, 5361. doi: 10.1111/j.1469-7610.2006.01634.x

Kearney, C. A. (2002). Identifying the function of school refusal behavior: A revision of the School Refusal Assessment Scale. Journal of Psychopathology and Behavioral Assessment, vol.24, n.4, 235-245. doi: 10.1023/A:1020774932043

Kearney, C. A. (2001). School refusal behavior in youth: A functional approach to assessment and treatment. Washington, DC: American Psychological Association.

Kearney, C. A. y Albano, A. M. (2004). The functional profiles of school refusal behavior. Behavior Modification, vol.28, n.1, 147-161. doi: 10.1177/0145445503259263

Kearney, C. A. y Bates, M. (2005). Adressing School Refusal Behavior: Suggestions for Frontline Professionals. Children \& Schools, vol.27, n.4, 207-216. doi: 10.1093/cs/27.4.207

Kearney, C. A. y Graczyk, P. (2014). A response to intevention model to promote school attendance and decrease school absenteeism. Child \&Youth Care Forum, vol.43, n.1, 1-25. doi: 10.1007/ s10566-013-9222-1

Kearney, C. A., Lemos, A. y Silverman, J. (2006). School refusal behavior. En R. B. Mennuti, A. Freeman, y R.W. Christner (Eds.), Cognitive-behavioral interventions in educational settings: A handbook for practice (pp. 89-105). New York: Brunner-Routledge.

Kearney, C. A., Lemos, A. y Silverman, J. (2004). The functional assessment of school refusal behavior. American Journal of Family Therapy, vol.5, n.3, 275-283.

Kearney, C. A. y Silverman, W. (1996). The evolution and reconciliation of taxonomic strategies for school refusal behavior. Clinical Psychology: Science and Practice, vol.3, n.4, 339-354. doi: 10.1111/j.1468-2850.1996.tb00087.x

Kearney, C. A. y Spear, M. (2014). School refusal behavior. En L. Grossman y S. Walfish (Eds.), Translating psychological research into practice (pp. 83-85). Washington, DC: American Psychological Association.

Kearney, C. A., Turner, D. y Gauger, M. (2010). School refusal behavior. En I. Weiner y E. Craighead (Eds.), Corsini's encyclopedia of psychology (4th ed.) (pp. 1517-1519). New York: Wiley.

King, N. J., Ollendick, T. H. y Tonge, B. J. (1995). School refusal: Assessment and treatment. Boston: Allyn \& Bacon.

Lagos-San Martín, N., Gómez-Núñez, M.I., Gonzálvez, C., Vicent, M. y Gomis, N. (2014). Relación entre atribuciones de éxito y fracaso académico en el área de lengua y rechazo escolar en una muestra de estudiantes chilenos de educación secundaria. International Journal of Developmental and Educational Psychology (INFAD Revista de Psicología), vol.1, n.4, 477-486.

MIDEPLAN. (2005). Adolescentes y jóvenes que abandonan sus estudios antes de finalizar la enseñanza media: Principales tendencias (documento elaborado por Melis, F., Díaz, R. y Palma, A. de la División Social de MIDEPLAN, Chile). Recuperado el 18 de octubre de 2014 de http:// www.oei.es/quipu/chile/desercionescolar.pdf

Mihalas, S. (2014). School refusal behavior. En L. Grossman y S. Walfish (Eds.), Translating psychological research into practice (pp. 85-88). Washington, DC: American Psychological Association.

Ollendick, T. H. y Mayer, J. A. (1984). School phobia. En S.M. Turner (Ed.), Behavioral theories and treatment of anxiety (pp. 367-411). Nueva York: Plenum.

Pina, A. A., Zerr. A. A., Gonzales, N. A. y Ortiz, C. D. (2009). Psychosocial interventions for school refusal behavior in children and adolescents. Child Development Perspectives, vol.3, n.1, 11-20.

Sewell, J. (2008). School refusal. Australian Family Physician, vol.37, n.6, 406-408.

Teasley, M. L. (2004). Absenteeism and Truancy: Risk, Protection, and Best Practice Implications for School Social Workers. Children \& Schools, vol.26, n.2, 117-128. doi: 10.1093/cs/26.2.117

Thomas, J. M., Lemieux, C. M., Rhodes, J. L. y Ashbaugh-Vlosky, D. (2011). Early truancy intervention: Results of an evaluation using a regression discontinuity design. Children and 
Youth Services Review, vol.33, n.9, 1563-1572. doi: 10.1016/j.childyouth.2011.03.021

Tolin, D. F., Whiting, S., Maltby, N., Diefenbach, G. J., Lothstein, M. A., Hardcastle, S. y Gray, K. (2009). Intensive (daily) behavior therapy for school refusal: A multiple baseline case series. Cognitive and Behavioral Practice, vol.16, n.3, 332-344. doi: 10.1016/j.cbpra.2009.02.003

Wilkins, J. (2008). School characteristics that influence student attendance: Experiences of students in a school avoidance program. The High School Journal, vol.91, n.3, 12-24. doi: 10.1353/ hsj.2008.0005

Wimmer, M. (2008). School Refusal. Principal Leadership, vol.8, n.8, 10-14.

Wijetunge, G. S. y Lakmini, W. D. (2011). School refusal in children and adolescents. Journal of Child Health, vol.40, n.3, 128-131. doi: http://dx.doi.org/10.4038/sljch.v40i3.3511 
